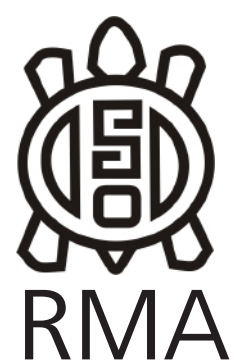

Dossier

\title{
Tafonomía de restos óseos provenientes de egagrópilas de Coragyps atratus (jote de cabeza negra) en el Noroeste de la Patagonia argentina
}

\author{
Fernando Ballejo *, Fernando J. Fernández ** y Luciano J. M. De Santis ***
}

*Comisión de Investigaciones Científicas. Cátedra de Anatomía Comparada. Facultad de Ciencias Naturales y Museo, Universidad Nacional de La Plata. E-mail: fernandoballejo@ hotmail.com. ${ }^{*}$ CONICET, Cátedra de Anatomía Comparada. Facultad de Ciencias Naturales y Museo. Universidad Nacional de La Plata. E-mail: fernandezf77@yahoo. com.ar. ${ }^{* * *}$ Cátedra de Anatomía Comparada. Facultad de Ciencias Naturales y Museo. Universidad Nacional de La Plata. E-mail: desantis@museo.fcnym.unlp.edu.ar

\begin{abstract}
Resumen
Existen diversos estudios actualísticos en tafonomía sobre restos de presas de diferentes especies de aves raptoras. Sin embargo, este tipo de trabajos en aves carroñeras ha sido escasamente considerado hasta el momento en Argentina, a pesar que ellas cumplen un papel fundamental en la alteración y transporte de cadáveres. Coragyps atratus (jote de cabeza negra) tiene la particularidad de formar posaderos comunales cercanos a áreas de actividades humanas y de alimentarse de animales de todos los tamaños, por lo que pueden interferir en la formación de sitios arqueológicos. El objetivo de este trabajo es realizar un análisis tafonómico sobre los restos óseos recuperados de egagrópilas de C. atratus en el Noroeste patagónico, con el fin de generar herramientas que permitan identificar a estas aves como posibles agentes acumuladores. Se recolectaron 469 egagrópilas distribuidas en tres posaderos del Noroeste patagónico. Se identificaron los elementos recuperados y se evaluaron los grados de alteraciones por ácidos gástricos. Lepus europaeus y Ovis aries dominaron las muestras. Los elementos más representativos pertenecen al autopodio, principalmente falanges. Todos ellos muestran signos de digestión, que van desde porosidades superficiales, perforaciones y fracturas, con la desaparición de la médula en el caso de varias falanges; denotando diferencias en relación al tamaño de la presa consumida.
\end{abstract}

Palabras claves: Coragyps atratus; tafonomía; carroña; zooarqueología.

Taphonomy of bones remains from pellets of Coragyps atratus (black vulture) in the northwest of the argentinian Patagonia

\begin{abstract}
There are several actualistic taphonomic studies regarding different species of birds of prey. However, the studies focus on scavenger birds have been scarcely considered in Argentina, despite the fact that they play a fundamental role in the alteration and transportation of carcasses. A peculiar characteristic of Coragyps atratus (black vulture) is that it builds communal roosts near places where human activities are developed and it feeds on animals of varying size, which may interfere with the formation of archaeological sites. The aim of this study is to perform a taphonomic analysis of the bones remains retrieved from pellets of C. atratus in the Northwest of Patagonia, with the purpose of creating tools that allow the identification of these birds as possible accumulator agents. 469 pellets, distributed in the three roosts of the patagonic norhtwest, were gathered. The elements retrieved were identified and the degrees of alterations caused by gastric acids were evaluated. Lepus europaeus and Ovis aries predominate in the samples. The main elements belong to the articular bones, mainly phalanges. They all show signs of digestion, from surface porosities to perforations and fractures, and in the case of several phalanges, the disappearance of marrow, denoting differences regarding the size of the ingested prey.
\end{abstract}

Keywords: Coragyps atratus; taphonomy; carrion; zooarchaeology.

Coragyps atratus (jote cabeza negra) es un ave perteneciente al Orden Ciconiformes; Familia Cathartidae, se distribuye desde el sudeste de Estados Unidos hasta la provincia de Chubut en la Argentina y habita en multiplicidad de ambientes (Del Hoyo et al. 1994). Los trabajos sobre su dieta describen a estas aves como carroñeras (DeVault et al. 2004; Kelly et al. 2007; Yahner et al. 1990), las cuales se alimentan tanto de animales domésticos como silvestres, incluyendo aves, reptiles, mamíferos, insectos, vegetales y hasta materiales no biológicos (Kelly et al. 2007; Thomaides et al. 1989). Debido a que la carroña es un recurso pobremente distribuido y efímero, éstas aves se agrupan en gran número formando posaderos comunales, generalmente 
en árboles, rocas y aleros, muchos de los cuales se encuentran en las inmediaciones de áreas antrópicas como basurales o campos ganaderos; bajo los cuales se concentran las egagrópilas y todos aquellos elementos no digeridos como huesos, dientes, plumas, pelos y estructuras córneas (Buckley 1996; Hiraldo et al. 1991; Kelly et al. 2007; Thomaides et al. 1989). En tal sentido, los restos óseos y dentarios pueden incorporarse al registro arqueológico o paleontológico formando acumulaciones, de difícil interpretación para los investigadores (Andrews 1990). Es por este motivo que un enfoque tafonómico actualístico será de suma importancia para establecer las características diagnósticas de las acumulaciones óseas de esta especie.

En los últimos tiempos se han realizado estudios tafonómicos sobre aves carroñeras en otras partes del mundo, destacándose los trabajos de Robert y Vigne (2002a, 2002b) sobre Gypaetus barbatus (quebranta huesos), Laudet y Selva (2005) sobre Corvus corax (cuervo) y Reeves (2009) sobre C. atratus y Cathartes

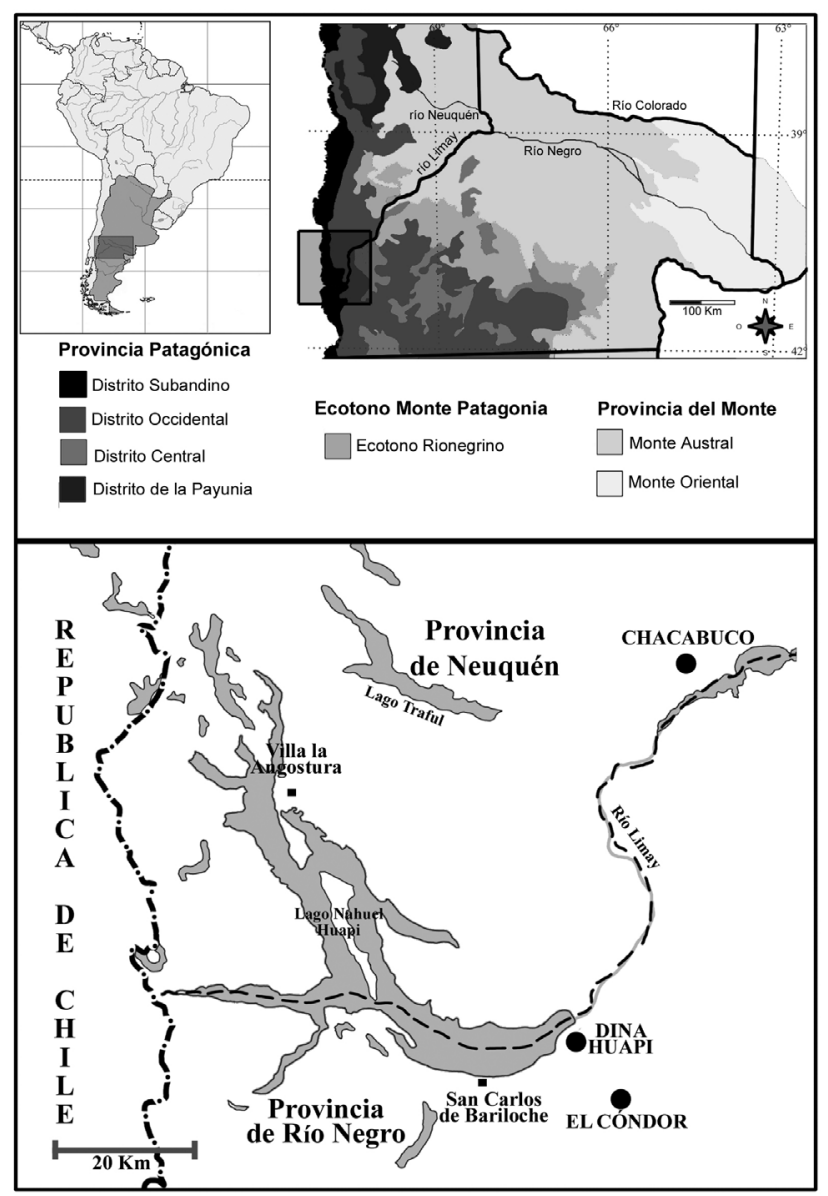

Figura 1. Mapa de Norpatagonia mostrando el área de estudio (arriba) y la ubicación de los posaderos de Coragyps atratus (abajo): Dina Huapi, El Cóndor y Chacabuco. Principales unidades fitogeográficas acordes a León et al. (1998).

Figure 1. Map of Northern Patagonia showing the study area (above) and locations of Coragyps atratus' roosts (below): Dina Huapi, The Condor and Chacabuco. Main phytogeographic units according to León et al. (1998). aura (jote cabeza colorada). Por su parte, en Argentina se han realizado análisis a partir de restos consumidos por Milvago chimango (chimango) (Gómez 2007) y sobre restos depredados por Caracara plancus (carancho) (Gómez 2007; Montalvo y Tallade 2009, 2010; Montalvo et al. 2011). No obstante, la participación de las aves carroñeras en la formación de los conjuntos arqueofaunísticos ha sido demostrada en estudios arqueológicos (e.g. Marín Arroyo et al. 2009; Robert y Vigne 2002a, 2002b; Robert y Reumer 2009).

Los registros zooarqueológicos en el noroeste de la provincia de Río Negro y sudoeste de la provincia de Neuquén son abundantes y mayormente presentan datos publicados sobre ensambles de micromamíferos y, en menor medida, sobre mamíferos medianos y grandes [Cueva Traful 1 (Pardiñas 1999, Pearson 1987; Pearson y Pearson 1993); Cueva Epullán Grande (Crivelli-Montero et al. 1996; Pardiñas 1999); El Trébol (Hajduk et al. 2004; Pardiñas y Teta 2008); Alero Arias (Pardiñas 1999; Teta et al. 2005); Casa de Piedra Ortega (Pardiñas 1999; Teta et al. 2005); Cueva Paredón Loncomán (Teta et al. 2005); Cueva Sarita I (Massoia 1982; Teta et al. 2005); Cueva Sarita II (Teta et al. 2005); Cueva Sarita IV (Teta et al. 2005); Piedra del Águila II (Menégaz 1996; Pardiñas 1999); Localidad Arqueológica Meliquina (Pérez y Batres 2008; Pérez et al. 2008)].

El principal objetivo de este trabajo es realizar un análisis tafonómico-actualístico de las acumulaciones óseas generadas por $C$. atratus en tres posaderos del Noroeste patagónico. De este modo, identificar tafonómicamente a C. atratus como agente acumulador para elaborar un modelo de interpretación de ensambles fósiles provenientes de sitios arqueológicos y paleontológicos.

\section{Área de estudio}

Se estudiaron muestras de egagrópilas recolectadas en tres posaderos ubicados en el Noroeste patagónico: uno en la provincia de Neuquén (estancia Chacabuco $\left.40^{\circ} 35^{\prime} 41^{\prime \prime S}, 70^{\circ} 58^{\prime} 16^{\prime \prime} \mathrm{O}\right)$ y dos en la provincia de Río

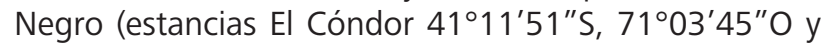
Dina Huapi $41^{\circ} 04^{\prime} 4^{\prime \prime}$ S, $71^{\circ} 09^{\prime} 27^{\prime \prime}$ ) (Figura 1).

Los tres sitios se encuentran en un área que incluye a las elevaciones mas altas de los Andes Norpatagónicos (2000-2300 msnm) y las serranías de la Precordillera Patagónica (1300-1800 msnm) (Giacosa y Marquez 1999; Giacosa et al. 2005). El área presenta un clima frío-templado, con inviernos fríos y abundantes nevadas y veranos más calidos y secos, con un importante gradiente de precipitaciones de Oeste-Este, influenciado por las masas de aire del Océano Pacifico y la barrera topográfica de la Cordillera de los Andes, lo que genera condiciones de mayor aridez hacia Este (Paruelo et al. 1998).

Desde una perspectiva fitogeográfica, el área se encuentra 
dentro de la Provincia Patagónica, Distrito Occidental (Figura 1), caracterizada por una estepa arbustivograminosa de Stipa speciosa, Stipa humilis, Senecio filaginoides y Mulinum spinosum (León et al. 1998).

Zoogeográficamente, el área forma parte del Dominio Andino Patagónico (Ringuelet 1961), caracterizado por la abundancia de aves rapaces tales como Geranoaetus melanoleucus (águila mora), Buteo polyosoma (aguilucho común), C. plancus, M. chimango, Tyto alba (lechuza de campanario), Bubo magellanicus (tucúquere) y de mamíferos carnívoros tales como Puma concolor (puma), Lycalopex culpaeus (zorro colorado), Lycalopex griseus (zorro gris chico), Lyncodon patagonicus (huroncito patagónico) y Conepatus chinga (zorrino común).

Finalmente, esta área está afectada por actividades humanas, tales como la ganadería y la caza deportiva (Lambertucci et al. 2009; Novillo y Ojeda 2008).

\section{Materiales y Métodos}

El posadero de $C$. atratus de Dina Huapi se encuentra dentro de un pinar de especies exóticas (Pinus ponderosa y Pseudotsuga menziesii) en el cerro Tortuga, a 2,3 km de un matadero, donde se faena ganado [Bos taurus (vaca) y Ovis aries (oveja)], cuyos desperdicios son aprovechados por estas aves (obs. pers.). Por otro lado, el posadero de la estancia Chacabuco está ubicado en un cordón de álamos próximos a la Ruta 40, donde se alimentan de animales muertos, fundamentalmente Lepus europaeus (liebre europea) (Donazar et al. 1993; Travaini et al. 1998). Por último, el posadero de C. atratus de la estancia El Cóndor consiste en un grupo reducido de árboles sobre la margen norte de un canal de agua. Dicha estancia es una de las principales productoras de lana en la región, contando con 30000 cabezas de ganado ovino, además de tener un coto de caza de Cervus elaphus (ciervo colorado).

Las acumulaciones de egagrópilas se encontraron en la base de los árboles mencionados, donde se posaban estas aves.

El método de cuantificación de individuos de $C$. atratus consistió en observar, mediante el uso de binoculares, a las aves ubicadas en los posaderos y volando en las inmediaciones de los mismos.

Se realizó una limpieza de los posaderos con el objetivo de recuperar las egagrópilas frescas y posteriormente, se recolectaron las mismas en tres campañas sucesivas, realizadas en los meses de mayo, septiembre y diciembre de 2010.

Los materiales óseos y dentarios fueron observados mediante lupa binocular (10-40 x) y algunos restos se seleccionaron para fotografiar con microscopio electrónico de barrido.
Con los restos recuperados se calculó el número de especímenes identificados por taxón (NISP), el número mínimo de elementos (MNE) y el número mínimo de individuos (MNI) por cada posadero. Para estudiar las frecuencias óseas se consideraron sólo los mamíferos por ser los más representativos en las muestras y se los dividió en tres categorías de tamaño: chicos (0-5 kg), medianos $(5-20 \mathrm{~kg})$ y grandes (mayor a $20 \mathrm{~kg}$ )

La metodología tradicionalmente utilizada para evaluar el grado de digestión causada por aves rapaces sobre los restos óseos de sus presas (principalmente en dientes, epífisis proximal de fémur y epífisis distal de húmero), es la propuesta por Andrews (1990) y Fernández-Jalvo y Andrews (1992). Sin embargo, debido a la baja representación de los elementos mencionados y a que el mayor número de ítems presa fueron mamíferos de mediano y gran tamaño, se siguió la propuesta metodológica de Robert y Vigne (2002a, 2002b) y Marín Arroyo et al. (2009) (Tabla 1). Estos autores desarrollaron una metodología de codificación, que permite clasificar los elementos del autopodio de mamíferos en relación al grado de alteración sufrido por los ácidos gástricos, con un gradiente de modificación, variando de 1 (marcas de digestión leves) hasta 5 o 9 (marcas de digestión fuerte), dependiendo del elemento. Para su notación, primero se refiere al número de falanges, a continuación del grado de alteración. A modo de ejemplo, el código 1.2 se refiere a la primera falange con porosidades en ambas carillas de articulación; el código 2.2 se refiere a la segunda falange y así sucesivamente. Esta metodología también se utilizó para analizar los elementos del basipodio (carpales y tarsales) y metapodio (metacarpos y metatarsos): el código 1 se refiere a elementos con erosión gástrica superficial; el 2 cuando presentan perforaciones en una de sus caras y faltan fragmentos de la médula; el 3 cuando las perforaciones se extienden al hueso entero y queda la cavidad medular abierta; en el código 4, el hueso está totalmente digerido y con los bordes adelgazados; por último el código 5 se refiere al estadio donde el elemento queda reducido a un pequeño fragmento casi no identificable.

La aplicación de estos índices para medir el grado de corrosión gástrica sólo se realizó para los elementos recuperados en Dina Huapi y Chacabuco, no así para los de El Cóndor, debido a que el número de los mismos fue muy bajo.

Finalmente, se utilizó el índice de abundancia relativa de los elementos esqueléticos formulado por Andrews (1990), R: MNEi / (EixMNI) x100, donde MNEi es el número mínimo de elementos esqueléticos particulares en la muestra y Ei es el número esperado de ese elemento esquelético en un individuo. Este índice se aplicó a cada dormidero por separado y luego se obtuvo un promedio de los mismos con el fin de generar gráficos que permitan la comparación con otros predadores. 


\section{PRIMER FALANGE}

1.1 Signos de ataque invisible al ojo desnudo.

1.2 Porosidad alrededor de las dos superficies articulares

1.3 Perforaciones bajo la inserción de tuberosidades, en general en cara dorsal-axial o palmar (parte proximal). O perforaciones en metáfisis de juveniles en la articulación proximal.

1.4 Desaparición de la superficie articular proximal.

1.5 Siguiendo el estadio 1.3, cuando la perforación genera la fractura de la falange. Sólo queda la porción articular proximal.

1.6 Desaparición de la superficie articular distal.

1.7 Sólo queda la polea distal.

\section{SEGUNDA FALANGE}

2.1 Signos de ataque invisible al ojo desnudo.

2.2 Porosidad alrededor de las dos caras articulares. Más visible en la vista palmar de la articulación distal.

2.3 Porosidad remarcada en las superficies articulares proximales.

2.4 Ambas carillas articulares se mantienen conectadas. Desaparece la cara axial o palmar de la columna que se adelgaza bajo la superficie articular proximal

2.5 Roturas bajo la superficie articular proximal. Sólo quedan la superficie articular distal y una porción de la columna disimétrica (más acentuada en la cara abaxial)

2.6 Sólo queda la porción distal.

2.7 Con una acentuación disimétrica y pérdida de la polea

2.8 Siguiendo al anterior, sólo queda la articulación proximal.

2.9 Articulación proximal además de la columna asimétrica

\section{TERCERA FALANGE}

3.1 Signos de ataque invisibles al ojo desnudo

3.2 Porosidad (borde dorsal) sobre o por debajo del proceso extensorio hasta el borde palmar, sólo en la cara axial. Amplitud del foramen vascular en dicha cara.

3.3 Falanges juveniles atacados en la superficie articular proximal.

3.4 Ambas carillas articulares se mantienen conectadas. Con más perforaciones del hueso compacto en el borde dorsal como en la cara axial y/o ataque sobre en ángulo palmar y superficie articular proximal.

3.5 Después de la rotura o disolución de la superficie articular proximal, quedan sólo el ápice, un fragmento plantar y una porción de la cara abaxial (disimétria).

3.6 Si la porosidad está también en el ápice, esta desaparece y sólo queda la superficie de articulación proximal más un fragmento columnar.

3.7 Si se rompe, sólo quedan la superficie articular proximal más una porción de la cara abaxial. Lo mismo se aplica si el ataque es simultáneo en el ápice.

3.8 Sólo queda la suela.

3.9 Entre estados 3.4 y 3.5

Tabla 1. Codificación de la intensidad de marcas de digestión en falanges (traducido al español de Marin Arroyo 2009: 288).

Table 1. Codification of the digestive marks on phalanges (according to Marin Arroyo 2009: 288).

\section{Resultados}

Se cuantificaron 45 individuos de C. atratus en la estancia Chacabuco, 130 en Dina Huapi y 65 en la estancia El Cóndor.

Se obtuvieron 469 egagrópilas, de las cuales 176 se recolectaron en Chacabuco, 193 en Dina Huapi y 100 en la estancia El Cóndor. El 43,9\% contenía elementos óseos y dentarios.

En conjunto se registraron 12 taxones (NISP=713, $\mathrm{MNE}=435, \mathrm{MNI}=24)$. L. europaeus y 0 . aries dominaron las muestras, seguidos por mamíferos indeterminados, además de dasipódidos, C. elaphus, Conepatus sp. (zorrino), Reithrodon auritus (rata conejo) y Myocastor coypus (coipo). Asimismo, se recuperaron restos indeterminados de peces, reptiles y aves (Tabla 2).

Como se ilustra en la Tabla 3, los valores de abundancia relativa de elementos óseos y dentarios de mamíferos muestran una representación mayoritaria de elementos del autopodio (42,8\% del total), principalmente falanges. Las terceras y primeras falanges son las más representativas, seguidas por fragmentos del metapodio, de los cuales el $18 \%$ del total correspondían a epífisis distales de individuos juveniles de 0 . aries. Cabe destacar la notable cantidad de elementos indeterminados (33,9\%).

Todos los elementos presentaron signos de digestión gástrica, sin embargo se observó una diferenciación en relación al tamaño de los mismos (Tabla 4).

Las terceras falanges de los mamíferos grandes de Dina Huapi fueron las más afectadas (Figura 2A), debido a que el $55 \%$ de las mismas presentaron importantes perforaciones y fracturas, principalmente en sus superficies dorsales, dejando la médula expuesta o ausente (Código 3.4). Por otro lado, la mayoría de las terceras falanges de los mamíferos medianos de Chacabuco (lugar donde estuvieron mejor representados), exhibieron menores niveles de corrosión digestiva, mostrando porosidades superficiales, principalmente localizadas en las regiones proximales (Código 3.3), (Figura 2B). Por otra parte, se registraron diferencias en los niveles de corrosión digestiva en las primeras falanges en relación a los posaderos estudiados y a las categorías de tamaño. En Dina Huapi, el 54\% de las primeras falanges pertenecientes a mamíferos grandes exhibieron perforaciones, ubicadas generalmente debajo de las tuberosidades o a lo largo de la diáfisis, dejando la falange completamente hueca (Código 1.3) (Figura 2C); sin embargo, los porcentajes más altos (75\% y $36 \%$ ) referidos a los grados de corrosión en las primeras falanges de mamíferos medianos de Dina Huapi y Chacabuco, mostraron un grado de corrosión menor que los encontrados en mamíferos grandes, ya que evidenciaron porosidades sólo en las caras articulares (Código 1.2). Sólo el $16 \%$ de primeras falanges de 


\begin{tabular}{lcccccccccccc}
\hline & \multicolumn{3}{c}{ DINA HUAPI } & \multicolumn{3}{c}{ CHACABUCO } & \multicolumn{2}{c}{ EL CÓNDOR } & \multicolumn{3}{c}{ TOTAL } \\
\hline Osteichthyes indet. & 56 & 37 & 1 & 5 & 5 & 1 & - & - & - & 61 & 42 & 2 \\
Reptilia indet. & - & - & - & 1 & 1 & 1 & - & - & - & 1 & 1 & 1 \\
Aves indet. & 1 & - & - & 15 & 15 & 1 & - & - & - & 16 & 15 & 1 \\
Mammalia indet. & 30 & 4 & - & 196 & 17 & - & 13 & 2 & - & 242 & 23 & - \\
Ovis aries & 66 & 58 & 3 & - & - & - & 2 & 2 & 1 & 68 & 60 & 4 \\
Cervus elaphus & 1 & 1 & 1 & - & - & - & - & - & - & 1 & 1 & 1 \\
Conepatus sp. & - & - & - & 32 & 29 & 1 & - & - & - & 32 & 29 & 2 \\
Lepus europaeus & 9 & 9 & 1 & 186 & 164 & 4 & 13 & 12 & 1 & 208 & 185 & 6 \\
Dasypodidae indet. & - & - & - & 3 & 3 & 1 & - & - & - & 3 & 3 & 1 \\
Rodentia indet. & - & - & - & 17 & 17 & 1 & - & - & - & 48 & 46 & 3 \\
Reithrodon auritus & 25 & 22 & 1 & - & - & - & 7 & 7 & 1 & 32 & 29 & 2 \\
Myocastor coypus & - & - & - & - & - & - & 1 & 1 & 1 & 1 & 1 & 1 \\
Total & 188 & 131 & 7 & 455 & 251 & 11 & 36 & 24 & 4 & 713 & 435 & 24 \\
\hline
\end{tabular}

Tabla 2. Composición taxonómica de los ensambles de vertebrados para cada dormidero y los valores totales (expresados en NISP, MNE y MNI).

Table 2. Taxonomic composition of the vertebrate samples for each roosts and total values (expressed in NISP, MNE and MNI).

Tabla 3. Número mínimo de elementos (MNE) y abundancia relativa (R\%) en los ensambles de mamíferos chicos (Ch.), medianos (Med.) y grandes (Gr) de los tres dormideros estudiados.

Table 3. Minimum number of elements (MNE) and relative abundance (R\%) in the small, medium, and large mammal assemblages from the three roosts studied.

\begin{tabular}{|c|c|c|c|c|c|c|c|c|c|c|c|c|c|c|c|c|}
\hline \multirow{3}{*}{ Códigos } & \multicolumn{6}{|c|}{ DINA HUAPI } & \multicolumn{4}{|c|}{ CHACABUCO } & \multicolumn{6}{|c|}{ EL CÓNDOR } \\
\hline & \multicolumn{2}{|c|}{ Ch. MNI:1 } & \multicolumn{2}{|c|}{ Med. MNI:1 } & \multicolumn{2}{|c|}{ Gr. MNI:4 } & \multicolumn{2}{|c|}{ Ch. MNI:2 } & \multicolumn{2}{|c|}{ Med.MNI:4 } & \multicolumn{2}{|c|}{ Ch. MNI:2 } & \multicolumn{2}{|c|}{ Med. MNI:1 } & \multicolumn{2}{|c|}{ Gr. MNI:1 } \\
\hline & MNE & $\mathrm{R} \%$ & MNE & $\mathrm{R} \%$ & MNE & $\mathrm{R} \%$ & MNE & $\mathrm{R} \%$ & MNE & $\mathrm{R} \%$ & MNE & $\mathrm{R} \%$ & MNE & $\mathrm{R} \%$ & MNE & $\mathrm{R} \%$ \\
\hline $\begin{array}{l}\text { Fragmento } \\
\text { del cráneo }\end{array}$ & 1 & 100 & - & - & 3 & 75 & 1 & 50 & 1 & 25 & 1 & 50 & - & - & - & - \\
\hline Mandíbula & - & - & - & - & 2 & 25 & - & - & - & - & - & - & - & - & - & - \\
\hline Incisivos & 1 & 25 & - & - & 2 & 6,25 & 1 & 12,5 & - & - & - & - & - & - & - & - \\
\hline Molares & 2 & 16,66 & - & - & 4 & 4,16 & - & - & - & - & 1 & 8,33 & - & - & - & - \\
\hline Vértebra & - & - & 1 & 3,8 & - & - & 3 & 5,76 & 15 & 14,4 & - & - & - & - & 1 & 3,8 \\
\hline Costillas & - & - & - & - & 6 & 6,25 & 2 & 4,16 & 6 & 6,25 & - & - & - & - & - & - \\
\hline Escápula & 1 & 50 & - & - & - & - & - & - & - & - & - & - & 1 & 50 & - & - \\
\hline Húmero & 1 & 50 & - & - & - & - & - & - & - & - & - & - & - & - & - & - \\
\hline Cúbito & - & - & - & - & - & - & 2 & 50 & - & - & - & - & - & - & - & - \\
\hline Fémur & 1 & 50 & - & - & - & - & 1 & 25 & 1 & 12,5 & - & - & - & - & - & - \\
\hline Peroné & - & - & - & - & - & - & - & - & 2 & 25 & - & - & - & - & - & - \\
\hline Rótula & - & - & - & - & - & - & 1 & 25 & 1 & 12,5 & - & - & - & - & - & - \\
\hline Tibia & 2 & 100 & - & - & - & - & 1 & 25 & 1 & 12,5 & - & - & - & - & - & - \\
\hline $\begin{array}{l}\text { Autopodio } \\
\text { fusionado }\end{array}$ & 1 & 25 & - & - & - & - & 1 & 12,5 & 1 & 6,25 & - & - & - & - & - & - \\
\hline $\begin{array}{l}\text { Basipodio } \\
\text { indet. }\end{array}$ & - & - & - & - & - & - & - & - & 2 & 2,1 & - & - & - & - & - & - \\
\hline Calcáneo & 1 & 50 & - & - & - & - & 3 & 75 & 6 & 75 & - & - & - & - & - & - \\
\hline Astrágalo & & & - & - & - & - & 1 & 25 & 1 & 12,5 & - & - & - & - & - & - \\
\hline Metapodio & 5 & 25 & - & - & 11 & 34,37 & 11 & 27,5 & 30 & 37,5 & 3 & 15 & 1 & 5 & - & - \\
\hline $1^{\text {a }}$ falange & - & - & 2 & 10 & 24 & 75 & 4 & 10 & 33 & 41,2 & 3 & 15 & 3 & 15 & 1 & 12,5 \\
\hline $2^{a}$ falange & - & - & 3 & 15 & 6 & 18.75 & 5 & 12,5 & 19 & 23,7 & - & - & 2 & 10 & - & - \\
\hline $3^{a}$ falange & 7 & 35 & 3 & 15 & 8 & 25 & 10 & 15 & 63 & 78,7 & - & - & 4 & 20 & - & - \\
\hline
\end{tabular}

mamíferos medianos tienen importantes perforaciones (Figura 2D), fracturas y pérdidas de sus epífisis distales.

Cabe destacar el alto porcentaje de falanges con las médulas totalmente digeridas, siendo el $83,8 \%$ para las falanges de $O$. aries y $31,2 \%$ para las de $L$. europaeus.

Finalmente, resulta interesante destacar el registro de placas óseas de dasipódidos y un molar del roedor cricétido $R$. auritus con altos grados de corrosión gástrica. Por un lado, las placas exhibieron un fuerte poseado de las superficies y los bordes redondeados (Figura 2E) y por el otro, el molar mostró las superficies oclusales redondeadas y el esmalte está prácticamente ausente, dejando la dentina expuesta (Figura 2F). 


\begin{tabular}{|c|c|c|c|c|c|c|c|c|c|c|c|c|c|c|c|c|c|c|c|c|c|c|c|c|c|c|c|}
\hline & \multicolumn{9}{|c|}{ CHACABUCO } & \multicolumn{18}{|c|}{ DINA HUAPI } \\
\hline & \multicolumn{9}{|c|}{ Mamíferos medianos } & \multicolumn{9}{|c|}{ Mamíferos grandes } & \multicolumn{9}{|c|}{ Mamíferos medianos } \\
\hline & 1 & 2 & 3 & 4 & 5 & 6 & 7 & 8 & 9 & 1 & 2 & 3 & 4 & 5 & 6 & 7 & 8 & 9 & 1 & 2 & 3 & 4 & 5 & 6 & 7 & 8 & 9 \\
\hline $1^{\text {a }}$ Falange & 2 & 36 & 16 & 29 & 5 & - & 11 & - & - & - & 4 & 54 & 29 & 8 & - & 4 & - & - & - & 75 & - & 25 & - & - & - & - & - \\
\hline $2^{\text {a }}$ Falange & - & 67 & 10 & 5 & 5 & 14 & - & - & - & - & 40 & 20 & - & - & - & - & - & 40 & - & - & - & 66 & 33 & - & - & - & - \\
\hline $3^{a}$ Falange & - & 26 & 70 & - & 4 & - & - & - & - & - & - & - & 55 & - & 11 & 22 & - & 11 & - & 33 & 33 & 33 & - & - & - & - & - \\
\hline $\begin{array}{l}\text { Basipodio } \\
\text { indet. }\end{array}$ & 50 & 50 & - & - & - & - & - & - & - & - & - & - & - & - & - & - & - & - & - & - & - & - & - & - & - & - & - \\
\hline Astrágalo & - & 50 & 50 & - & - & - & - & - & - & - & - & - & - & - & - & - & - & - & - & - & - & - & - & - & - & - & - \\
\hline Calcáneo & 40 & 20 & 40 & - & - & - & - & - & - & - & - & - & - & - & - & - & - & - & - & - & - & - & - & - & - & - & - \\
\hline Metapodio & 22 & 10 & 37 & 27 & 3 & - & - & - & - & 66 & 16 & 16 & - & - & - & - & - & - & - & - & - & - & - & - & - & - & - \\
\hline
\end{tabular}

Tabla 4. Representación porcentual de elementos del autopodio de mamíferos medianos y grandes encontrados en los dormideros de Chacabuco y Dina Huapi para cada categoría de digestión (sensu Robert y Vigne 2002a, 2002b, Marín Arroyo et al. 2009).

Table 4. Percentage of autopod elements of the medium and large mammals found in Chacabuco and Dina Huapi roosts for each digestive category (sensu Robert and Vigne 2002a, 2002b, Marín Arroyo et al. 2009).

\section{Discusión y conclusiones}

En las muestras de C. atratus se observaron similitudes en los niveles de corrosión digestiva con ensambles generados por mamíferos carnívoros. En tal sentido, Martin y Borrero (1997) encontraron acumulaciones óseas asociadas a fecas de $P$. concolor en un dormidero, donde todas ellas tenían evidencias de corrosión digestiva y pertenecían a $O$. aries, L. europaeus y roedores sigmodontinos. Por otro lado, Montalvo et al. (2007) analizaron 76 fecas de pumas, con dominancia de roedores en la muestra, seguidos de carnívoros, artiodáctilos, dasipódidos y lagomorfos. Los autores mencionados observaron que el material óseo de los animales grandes estaba compuesto por fragmentos no identificables debido a una fragmentación ósea extensiva con evidencias de una digestión fuerte, con adelgazamientos, áreas prominentes pulidas y con orificios. No obstante, una diferencia importante entre los restos óseos acumulados por C. atratus y aquellos depositados por mamíferos carnívoros, radica en que éstos últimos ocasionan crenulaciones y orificios en los huesos por la acción masticatoria.

Se encontraron diferencias importantes en los taxones consumidos de los tres dormideros estudiados, lo que influye sobre las abundancias relativas de los elementos recuperados (Tabla 2 y 3). En este sentido, el posadero de Dina Huapi cuenta principalmente con conjuntos óseos de $O$. aries, que debido a su gran tamaño dificulta la ingestión de partes esqueletales; razón por la cual, los elementos de menor tamaño (i.e., autopodio), son los más representativos en esa muestra. El posadero de la estancia Chacabuco no cuenta con elementos óseos de mamíferos grandes, siendo $L$. europaeus la especie mejor representada, incorporando al registro elementos pertenecientes a las porciones más proximales de los miembros. Esto se observó en mayor proporción en mamíferos chicos, donde se cuentan, entre otros, elementos del estilopodio y zeugopodio.
La Figura 3 representa los valores promedios de abundancia relativa de mamíferos chicos y medianos, comparados con los datos obtenidos de otros predadores, cuyas presas coinciden con las categorías de tamaño consideradas (Andrews 1990; Gómez y Kaufmann 2007; Hockett 1995; Montalvo et al. 2007; Lloveras et al. 2008; Lloveras et al. 2011). En tal sentido, la abundancia relativa de partes esqueletales de las dos muestras de mamíferos chicos y medianos depositados por $C$. atratus presentaron patrones particulares que las diferencian de otros depredadores.

Asimismo, la elevada abundancia de elementos del autopodio de los mamíferos medianos y grandes acumulados por C. atratus (Tabla 3) es semejante a los agregados óseos de artiodáctilos generados por $L$. culpaeus y L. griseus (Mondini 1995).

Los resultados arribados en este trabajo muestran similitudes con aquellos obtenidos por Robert y Vigne (2002a, 2002b) sobre un estudio tafonómico actualístico de G. barbatus. En líneas generales esta ave presenta hábitos alimenticios similares a C. atratus, consumiendo principalmente carroña de diversos animales de mediano y gran porte, aunque con la diferencia que su dieta incluye una alta cantidad de huesos (70\% a 90\%), de donde obtienen sustancias orgánicas, especialmente lípidos. Robert y Vigne (2002a) analizaron 871 elementos óseos obtenidos de egagrópilas y describieron las acumulaciones óseas generadas por estas aves en cuatro rasgos: 1) la muestra está dominada por ungulados de tamaño mediano; 2) las frecuencias de las partes esqueletales están altamente desbalanceadas a favor de la tercera falange y secundariamente a la segunda y primera falange, además de la escápula y huesos sesamoideos; 3) marcas de digestión fuerte afectan a la mayor parte de la porción proximal de la segunda y tercera falange; 4) escasos fragmentos óseos indeterminados. 

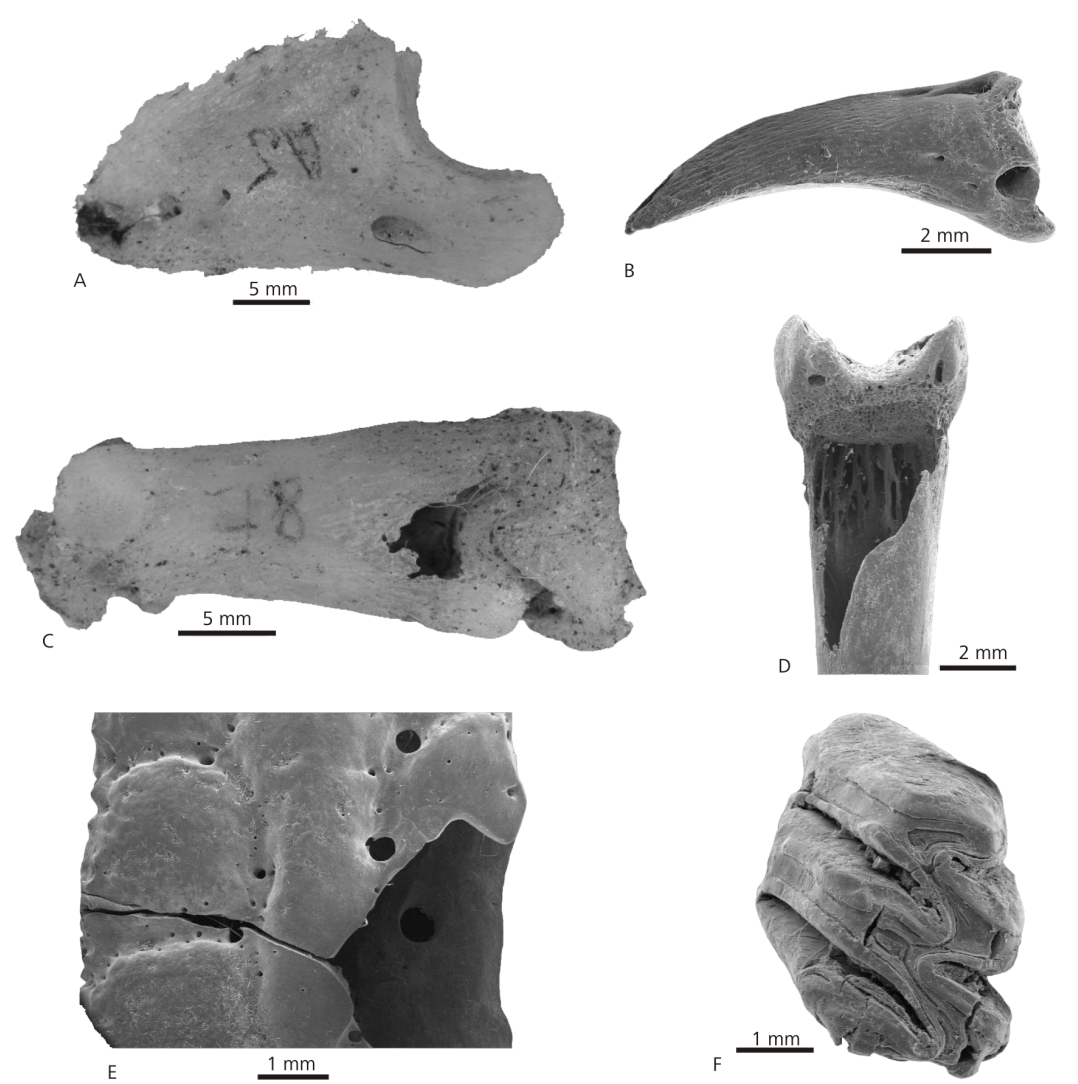

D
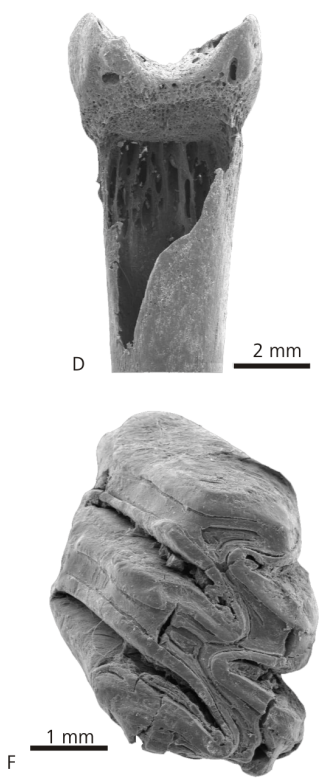

Figura 2. Ejemplos de marcas de digestión en diferentes tipos de huesos y taxones. A. Tercera falange de Ovis aries; B. Tercera falange de Lepus europaeus; C. Primera falange de O. aries; D. Primera falange de L. europaeus; E. Placa de Dasypodidae; F. Primer molar superior de Reithrodon auritus.

Figure 2. Examples of digestive marks at different kind of bones and taxa. A. Third phalange of Ovis aries; $B$. Third phalange of Lepus europaeus; $C$. First phalange of $\mathrm{O}$. aries; $D$. First phalange of $\mathrm{L}$. europaeus; E. Plate of Dasypodidae; F. First upper molar of Reithrodon auritus.

generados por un ave estrictamente carroñera - C. atratus- las cuales nunca habían sido consideradas en nuestro país a la hora de analizar un conjunto arqueofaunístico, a pesar de que existen varias especies de aves incluidas en este gremio [e.g., Vultur gryphus (cóndor andino), Cathartes burrovianus (jote cabeza amarilla), C. aura (jote cabeza colorada), Sarcoramphus papa (jote real)], que podrían producir acumulaciones de huesos y posteriormente incorporarse al registro arqueológico y paleontológico.
La representación de los ungulados en las acumulaciones óseas generadas por C. atratus, si bien no domina las muestras, presenta un porcentaje considerable (10\%). En este sentido, los elementos óseos de vertebrados de tamaño mediano como L. europaeus son los más representativos al considerar el total de las muestras $(30,5 \%)$. Sin embargo, se observó una importante variabilidad entre los posaderos estudiados. En Dina Huapi, los restos pertenecientes a 0 . aries $(35,1 \%)$ fueron los más representados, mientras que en El Cóndor y Chacabuco los restos de L. europaeus (36\% y 40,9\% respectivamente) fueron los más abundantes.

Por otro lado, la representación de falanges en las muestras es notable. Como ocurre con otras aves depredadoras y carroñeras tales como $G$. barbatus y C. plancus, la alta representación de elementos del autopodio de fauna mediana y grande, podría relacionarse con una mejor preservación por su tamaño y densidad ósea, por la selección del ave al ingerir ciertas porciones y descartar otras o por el aprovechamiento nutritivo de las médulas óseas (Montalvo y Tallade 2009; Robert y Vigne 2002a, 2002b). Esto está evidenciado por los altos porcentajes de elementos con la médula totalmente digerida encontrados en esta muestra. Asimismo, el grado menor de digestión en las terceras falanges de mamíferos medianos respecto de los mamíferos grandes, posiblemente se deba a que las mismas se encontraron dentro de los estuches córneos, favoreciendo su protección.

En este trabajo se abordaron distintos atributos sobre huesos, principalmente de mamíferos medianos y grandes,
Por el contrario, se han realizado numerosos trabajos actualísticos en mamíferos carnívoros, generadores de restos de mediano y gran tamaño (e.g., Andrews y Evans 1983; Borrero y Martín 1996; Borrero et al. 2005; Mondini 1995; Mondini y Muñoz 2008; Montalvo et al. 2007; Muñoz et al. 2008) y la participación de estos depredadores en la formación del registro arqueológico ha sido demostrada (e.g., Fernández et. at. 2011; Lyman 1994; Martin y Borrero 1997).

En síntesis, C. atratus genera acumulaciones caracterizadas por los siguientes rasgos: 1) las muestras están dominadas por vertebrados de mediano tamaño; 2) hay una alta representación de elementos del autopodio; 3) se evidencian marcas de digestión fuerte sobre las falanges que van desde porosidades superficiales, perforaciones y hasta fracturas y 4) existen diferencias en el grado de digestión entre los huesos de los distintos tipos de presas. Por otro lado, si bien la mayoría de los especímenes analizados en este trabajo pertenecen a especies de mamíferos introducidos que no se encuentran en el registro arqueológico prehispánico, los resultados obtenidos pueden ser comparados con especies autóctonas de tamaños similares.

La congregación de C. atratus en aleros, roquedales o árboles cercanos a zonas de actividad humana nos exige prestar especial atención a la interferencia que pueden causar como acumuladoras de restos óseos en sitios arqueológicos. A pesar que los elementos del autopodio no se caracterizan por ser aprovechados nutritivamente por los grupos humanos, dichas partes del miembro 


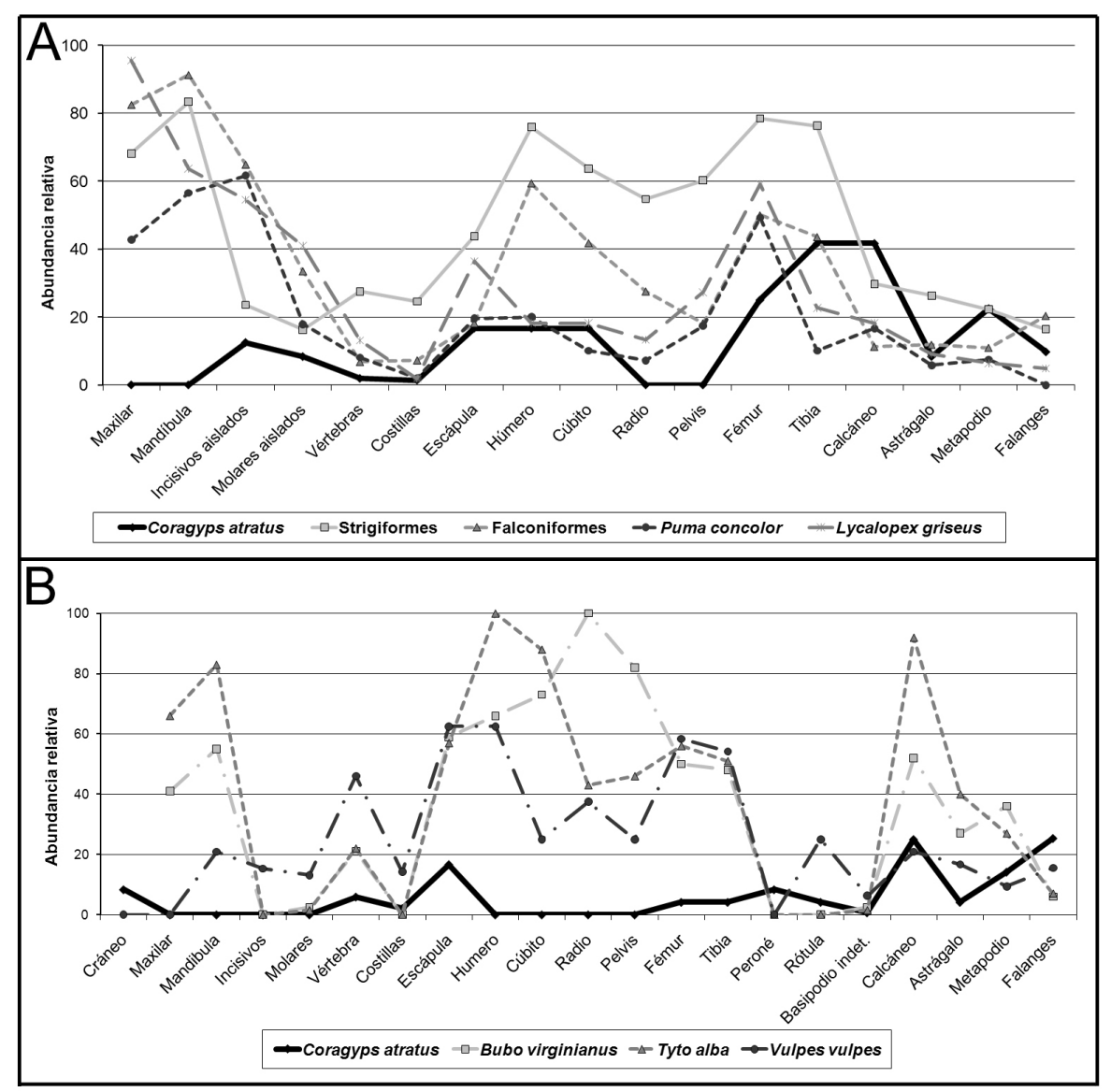

Figura 3. Valores de abundancia relativa de varios elementos anatómicos de mamíferos chicos (A) y mamíferos medianos (B), comparando las muestras de Coragyps atratus con otros depredadores: A [promedios de aves Strigiformes y Falconiformes (Andrews 1990); Puma concolor (Montalvo et al. 2007); Lycalopex griseus (Gómez y Kaufmann (2007).], B [Tyto alba y Bubo virginianus (Hockett 1995 y Lloveras 2011); Vulpes vulpes (Lloveras 2008)].

Figure 3. Relative abundance values for the various anatomical elements of the small mammals (A) and medium mammals (B), comparing the Coragyps atratus samples with others predators: A laverage of Strigiformes and Falconiformes birds (Andrews 1990); Puma concolor (Montalvo et al. 2007); Lycalopex griseus (Gómez and Kaufmann (2007).], B [Tyto alba and Bubo virginianus (Hockett 1995 and Lloveras 2011); Vulpes vulpes (Lloveras 2008)].

facilitan el acarreo de las porciones con mayor aporte nutritivo, además suelen quedar asociadas en el proceso de cuereo e incluso pueden ser trasladadas para su posterior uso como herramientas (Lyman 1994; Politis et al. 2011). En este sentido, resulta importante considerar la abundancia relativa de los elementos óseos recuperados y el grado de digestión de los mismos antes de hacer interpretaciones sobre el posible consumo por parte de mamíferos carnívoros o grupos humanos.

La Plata, 15 de Febrero de 2012

\section{Agradecimientos}

Luis del Papa y dos revisores anónimos realizaron comentarios y sugerencias constructivas a la primera versión del trabajo. María Clara Álvarez y Dan Rafuse nos invitaron a participar de este volumen. La Universidad Nacional de La Plata (Proyecto 11/N601) y la Comisión de Investigaciones Científicas de la provincia de Buenos Aires $(\mathrm{CIC})$ aportaron fondos para la concreción de este trabajo. Sergio Lambertucci ayudó en la localización de los posaderos; María del Mar Contaldi y Gonzalo Ignazi colaboraron en el campo. Carlos y Erna nos permitieron ingresar a sus estancias. A todas las personas e instituciones nombradas el reconocimiento de los autores, únicos responsables de los conceptos aquí vertidos.

\section{Bibliografía}

Andrews, P. 1990. Owls, caves and fossils. University of
Chicago Press, 231 pp., Londres.

Andrews, P. y E. M. Evans. 1983. Small mammal bone accumulations produced by mammalian carnivores. Paleobiology 9: 289-307.

Borrero, L. A., F. M. Martín. 1996. Gómez Otero, J., (Ed.), Tafonomía de carnívoros: un enfoque regional, 189-206. Arqueología, Sólo Patagonia. CENPAT (CONICET) Puerto Madryn.

Borrero, L. A., F. M. Martín., J. Vargas. 2005. Tafonomía de la interacción entre pumas y guanacos en el Parque Nacional Torres del Paine, Chile. Magallania 33: 95-114.

Buckley, N. J. 1996. Food finding and the influence of information local enhancement, and cummunal roosting on foranging success of north american vultures. The Auk 113(2): 473-488.

Crivelli-Montero, E. A., U. F. J Pardiñas, M. M. Fernández, M. Bogazzi, A. Chauvin, V. M. Fernández y M. J. Lezcano. 1996: La Cueva Epullán Grande (Provincia del Neuquén, Argentina) Informe de avance. Prehistoria 2: 185-240.

Del Hoyo, J., E Andrew y S. Jordi. 1994. Handbook of the birds of the world. Vol II: new world vultures to guinea fowl. 600 pp., Lynx edicions. Barcelona.

DeVault, T. L., Brisbin, I. L. y Rhodes, O. E. 2004. Factors 
influencing the acquisition of rodent carrion by vertebrate scavengers and decomposers. Canadian Journal of Zoology 82: 502-509.

Donazar, J. A., O. Ceballos, A. E. Travaini y F. Hiraldo. 1993. Roadside raptor surveys in the Argentinean Patagonia. J. Raptor Res. 27: 106-110.

Fernández, F. J., L. M. Del Papa, G. Moreira, L. Prates y L. J. M. De Santis. 2011. Small mammal remains recovered from two archaeological sites in the middleand lower Negro River valley (Late Holocene, Argentina): Taphonomic issues and paleoenvironmental implications. Quaternary international 245: 136-147.

Fernández-Jalvo, y P. Andrews. 1992. Small Mammal Taphonomy of Gran Dolina, Atapuerca (Burgos), Spain. Journal of Archaeological Science 19: 407-428.

Giacosa, R. E., J. C. Alfonso, N. Heredia y J. Paredes. 2005. Tertiary tectonics of the sub-Andean region of the North Patagonian Andes, southern central Andes of Argentina. J. S. Am. Earth Sci. 20: 157-170.

Giacosa, R. E. y M. J. Márquez. 2009. Jurásico y Cretácico de la Cordillera Patagónica septentrional y Precordillera Patagónica. Caminos, R. (Ed). Geología argentina. 17: 444-459, SEGEMAR, Buenos Aires.

Gómez, G. 2007. Predators categorization based on taphonomic analysis of micromammals bones: a comparison to proposed models. Gutierrez, M.A., Miotti, L., Barrientos, G., Mengoni, G. \& Salemme, M., eds. Taphonomy and Zooarchaeology in Argentina. pp. 89-103.

Gómez, G. N. y C. A. Kaufmann. 2007. Taphonomic analysis of Pseudalopex griseus (Gray, 1837) Scat assemblages and their archaeological implications. Journal of Taphonomy 5: 59-70

Hajduk, A., A. Albornoz y M. J. Lezcano. 2004. El "Mylodon" en el Patio de Atrás. Informe Preliminar Sobre los Trabajos en el Sitio El Trébol, Ejido Urbano de San Carlos de Bariloche, Provincia de Río Negro. In Contra Viento y Marea. M. T. Civalero, P. M. Fernández, and A. G. Guráieb, (eds). Arqueología de Patagonia. pp. 715-31. Sociedad Argentina de Antropología, Buenos Aires.

Hiraldo, F., Delibes M. y Donazar, J. A. 1991. Comparison of diets of Turkey Vultures in three regions of northern Mexico. Journal of Field Ornithology 62: 319-324.

Hockett, B. S., 1995. Comparison of leporid bones in raptor pellets, raptor nests, and archaeological sites in the Great Basin. North American Archaeologist 16: 223-238.

Kelly, N. E., D. W. Sparks, T. L. DeVault y O. E. Rhodes Jr. 2007. Diet of black and turkey vultures in a forested landscape. The Wilson Journal of Ornithology 119: 267-270.
Lambertucci S. A., K. L. Speziale, T. E. Roger y J. M. Morales. 2009. How do roads affect the habitat use of an assemblage of scavenging raptors?. Biodivers Conserv 18: 2063-2074.

Laudet F. y N. Selva. 2005. Ravens as small mammal bone accumulators: First taphonomic study on mammal remains in raven pellets. Palaeogeography, Palaeoclimatology, Palaeoecology 226: 272-286.

León, R. J., D. Bran, M Collantes, J. M. Paruelo y A. Soriano. 1998. Grandes unidades de vegetación de la Patagonia extra andina. Ecología Austral 8: 125-144.

Lloveras, L., M. Moreno Garcia, J. Nadal. 2008. Taphonomic study of leporid remains accumulated by the Spanish Imperial Eagle (Aquila adalberti). Geobios 41: $91-100$

Lloveras, L., M. Moreno Garcia, J. Nadal. 2011. Feeding the Foxes: An Experimental Study to Assess Their Taphonomic Signature on Leporid Remains. International Journal of Osteoarchaeology. DOI: 10.1002/oa.1280.

Lyman, R. L. 1994. Vertebrate Taphonomy. Cambridge University Press, Cambridge.

Marín Arroyo, A. B., P. Fosse y J. D. Vigne. 2009. Probable evidences of bone accumulation by Pleistocene bearded vulture at the archaeological site of El Mirón Cave (Spain). Journal of Archaeological Science 36: 284-296.

Martin, F. M. y L. A. Borrero. 1997. A Puma Lair in Southern Patagonia: Implications for the Archaeological Record. Current Anthropology 38: 453-461

Massoia, E. 1982. Restos de mamíferos recolectados en el paraje Paso de los Molles, Pilcaniyeu, Río Negro. Revista de Investigaciones Agropecuarias, INTA 7: 39-53.

Menégaz, A. 1996. Análisis del material funístico procedente del sitio Piedra del Aguila II (Neuquén, Argentina). Prehistoria 2: 147-171.

Mondini, M. N. 1995. Artiodactyl prey transport by foxes in puna rock shelters. Courrent Anthropology. 36 (3): 520-524.

Mondini, M. N. y S. Muñoz. 2008. Pumas as taphonomic agents: A comparative analysis of actualistic studies in the Neotropics. Quaternary International 180: 52-62.

Montalvo, C. I.; M. E. M. Pessino y V. H. Gonzalez. 2007. Taphonomic analysis of mammals remains eaten by pumas (Puma concolor Carnivora, Felidae). En central Argentina. Journal of Archaeological Science 34: 2151-2160.

Montalvo, C. I. y P. O. Tallade. 2009. Taphonomy of the Accumulations Produced by Caracara plancus 
(Falconidae). Analysis of Prey Remains and Pellets. Journal of Taphonomy 7: 235-248.

Montalvo, C. I. y P. O. Tallade. 2010. Análisis tafonómico de restos no ingeridos de roedores presa de Caracara plancus (Aves, Falconidae). De Nigris, M., P.M. Fernández, M. Giardina, A.F. Gil, M.A. Gutiérrez, A. Izeta, G. Neme y H.D. Yacobaccio (eds.), Zooarqueología a principios del siglo XXI: aportes teóricos, metodológicos y casos de estudio, 419-428 pp, Buenos Aires.

Montalvo C.; P. Tallade; F. J. Fernández, G. Moreira y L. De Santis. 2011. Pattern of damage done by Caracara plancus (Aves, Falconiformes) on bird bones. Journal of Archaeological Science 38: 3541-3548.

Muñoz, S., M. N. Mondini, V. Durán y A. Gasco. 2008. Los pumas (Puma concolor) como agentes tafonómicos. Análisis actualístico de un sitio de matanza en los Andes de Mendoza, Argentina. Geobios 41: 123-131.

Novillo, A. y R. A. Ojeda. (2008). The exotic mammals of Argentina. Biol. Invas. 10: 1333-1344.

Pardiñas, U.F.J. 1999. "Los roedores muroideos del Pleistoceno Tardio-Holoceno en la Región Pampeana (Sector Este) y Patagonia (República Argentina): aspectos taxonómicos, importancia bioestratigráfica y significación paleoambiental". Facultad de Ciencias Naturales y Museo. Universidad Nacional de La Plata, Argentina. 283 pp. Tesis Doctoral inédita.

Pardiñas, U. F. J. y P. Teta. 2008. Small mammals and paleoenvironments around the Pleistocene-Holocene boundary un Patagonia. Current Research in the Pleistocene 25: 30-32.

Paruelo, J. M., A. Beltran, E. Jobbagy, O. E. Sala y R. A. Golluscio (1998). The climate of Patagonia: general patterns and controls on biotic processes. Ecología Austral 8: 85-101.

Pearson, O. 1987. Mice and postglacial history of the Traful valley of Argentina. J. Mammalogy 68: 469-478.

Pearson, O., y A.K. Pearson. 1993. La fauna de mamíferos pequeños de Cueva Traful I, Argentina: Pasado y presente. Prehistoria 1: 211-224.

Pérez, A. y D. Batres. 2008. Los otros cazadores. Explotación de cérvidos en la Localidad Arqueológica Meliquina, Parque Nacional Lanín, República Argentina. J. Carlos Díez (ed). Zooarqueología hoy. Encuentros hispano-argentinos. Pp. 89-108. Universidad de Burgos. Burgos, España.
Pérez, A. E.; M. Smith, y E. Grillo. 2008. Implicancias tafonómicas de la composición faunística en la Localidad Arqueológica Meliquina, Parque Nacional Lanín, Pcia. de Neuquén, Argentina. RUNA 29: 79-99.

Politis G. G., L. Prates, M. L. Merino y M. F. Tognelli. 2011. Distribution parameters of guanaco (Lama guanicoe), pampas deer (Ozotoceros bezoarticus) and marsh deer (Blastocerus dichotomus) in Central Argentina: Archaeological and paleoenvironmental implications. Jounal of archaeological Science. 38 (7): 1405-1416

Reeves, N. M. 2009. Taphonomic effects of vulture scavenging. Journal of Forensic Sciences 54: 523-528.

Ringuelet, R. 1961. Rasgos fundamentales de la zoogeografía Argentina. Physis 22: 51-170.

Robert, I y J. D. Vigne. 2002a. The Bearded Vulture (Gypaetus barbatus) as an Accumulator of Archaeological Bones. Late Glacial Assemblages and Present-day Reference Data in Corsica (Western Mediterranean). Acta zoologica cracoviensia 45: 319-329.

Robert, I y J. D. Vigne. 2002b. Bearded Vulture Gypaetus barbatus contributions to the constitution of two different bone assemblages: Modern reference data and an archaeological example in Corsica. Journal of Archaeological Science 29: 763-777.

Robert, I. y J. W. F. Reumer. 2009. Taphonomic reinterpretation of a bone sample of endemic Pleistocene deer from Crete (Greece): osteoporosis versus regurgitation. Paleodiversity 2: 379-385.

Teta, P., A. Andrade y U. F. J. Pardiñas. 2005. Micromamíferos (Didelphimorphia y Rodentia) y paleoambientes del Holoceno tardío en la Patagonia noroccidental extraandina (Argentina). Archaeofauna, 11: 183-197.

Thomaides, C., R. Valdex, W. H. Reid y R. J. Raitt. 1989. Food habits of Turkey Vultures in west Texas. Journal of Raptor Research 23: 42-44.

Travaini, A., J. A. Donázar, A. Rodriguez, O. Ceballos, M. Funes, M. Delibes y F. Hiraldo. 1998. Use of european hare (Lepus europeus) carcasses by an avian cavenging assemblange in Patagonia. J. Zool. Lond. 246: 175-181.

Yahner, R. H., Storm, G. L, y Thompson, W. L. 1990. Winter diets of vultures in Pennsylvania and Maryland. Wilson Bulletin 102: 320-325. 